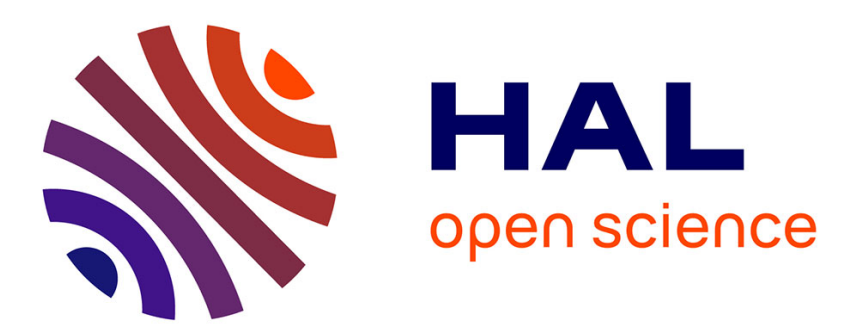

\title{
Molecular Simulations of Supercritical Fluid Permeation through Disordered Microporous Carbons
}

\author{
Alexandru Botan, Romain Vermorel, Franz-Josef Ulm, Roland J.-M. Pellenq
}

\section{To cite this version:}

Alexandru Botan, Romain Vermorel, Franz-Josef Ulm, Roland J.-M. Pellenq. Molecular Simulations of Supercritical Fluid Permeation through Disordered Microporous Carbons. Langmuir, 2013, 29 (32), pp.9985-9990. 10.1021/la402087r . hal-00856240

\section{HAL Id: hal-00856240 \\ https://hal.science/hal-00856240}

Submitted on 30 Aug 2013

HAL is a multi-disciplinary open access archive for the deposit and dissemination of scientific research documents, whether they are published or not. The documents may come from teaching and research institutions in France or abroad, or from public or private research centers.
L'archive ouverte pluridisciplinaire $\mathbf{H A L}$, est destinée au dépôt et à la diffusion de documents scientifiques de niveau recherche, publiés ou non, émanant des établissements d'enseignement et de recherche français ou étrangers, des laboratoires publics ou privés. 


\title{
Molecular simulations of supercritical fluid permeation through disordered microporous
}

\section{carbons}

Alexandru Boţan, ${ }^{*, \dagger}$ Romain Vermorel, ${ }^{\dagger, \S}$ Franz-Josef Ulm, ${ }^{\dagger, \ddagger}$ and Roland J.-M. Pelleng ${ }^{\dagger}+$, I

Department of Civil and Environmental Engineering, Massachusetts Institute of Technology, Cambridge, MA, USA, MSE2, UMI 3466 CNRS-MIT, Massachusetts Institute of Technology, Cambridge MA, USA, and CINaM, Aix Marseille University, 13288 Marseille, FRANCE

E-mail: botan@mit.edu

\begin{abstract}
Fluid transport through microporous carbon-based materials is inherent in numerous applications, ranging from gas separation by carbon molecular sieves to natural gas production from coal seams and gas shales. The present study investigates the steady-state permeation of supercritical methane in response to a constant cross-membrane pressure drop. We performed dual control volume grand canonical molecular dynamics (DCV-GCMD) simulations to mimic the conditions of actual permeation experiments. To overcome arbitrary assumptions regarding the investigated porous structures, the membranes were modeled after the CS1000a and CS1000 molecular models, representative of real microporous carbon materials. When

\footnotetext{
${ }^{*}$ To whom correspondence should be addressed

†IT

${ }^{\ddagger} \mathrm{MSE}^{2}$

"IINaM

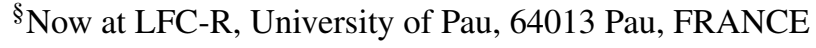


adsorption induced molecular trapping (AIMT) mechanisms are negligible, we show that the permeability of the microporous material, while not significantly sensitive to the pressure gradient, monotonically decreases with temperature and reservoirs pressures consistently with diffusion theory. On the other hand, when AIMT occurs, the permeability increases with temperature in agreement with experimental data found in the literature.

\section{Introduction}

The problem of fluid transfer through carbon-based disordered microporous materials is inherent in numerous filtration processes. Water and wastewater treatment, ${ }^{?}$ gas separation, ${ }^{?}$ energy storage, ${ }^{?}$ carbon dioxide sequestration or gas production from coal seams and gas shales fall in that category of applications for which the prediction of the permeability is of utmost practical importance. Aside from thermodynamical parameters, such as temperature, bulk fluid pressure and pressure gradient, other structural parameters greatly impact the permeability of microporous systems, namely the nature of the permeating fluid, the chemical composition of the solid and the texture of the porous network. Except for idealized porous structures, ${ }^{?} ?$ ? the effect of these factors is not analytically defined but can be investigated by experiments, including numerical ones. Molecular modeling has indeed been widely used to study permeation and selectivity in disordered microporous carbons assuming slit pore geometries. ? ? ? In such idealized porous structures, adsorption does not prevent the fluid molecules to diffuse through all the porous volume. This results in a decrease of the molar flux with temperature, consistently with the decrease of the interstitial concentration. In actual experiments however, measurements performed on real disordered microporous carbons reveal the opposite behavior.? ? Two different arguments may therefore be invoked to explain these discrepancies: firstly, simply accounting for a more realistic texture of the pore walls might imply additional effects not captured by regular slit pores models; On the other hand, single slit pore models might fail because they disregard the effects of constrictions as only one percolating pore is considered. ? ? The present work investigates both possibilities.

In this paper, we study the permeability of CS1000a and CS1000? as models for disordered mi- 
croporous carbons. Due to the presence of a wide percolating pore, the CS1000a molecular model can be thought of as a slit pore with enhanced pore walls texture. In contrast, the CS1000 exhibits a pore size distribution conducive to Adsorption Induced Molecular Trapping (AIMT) effects as a result of numerous pore constrictions. The steady-state permeation of a single component fluid through a disordered microporous membrane is addressed. We use dual control volume - grand canonical molecular dynamics (DCV-GCMD) to mimic the experimental conditions of supercritical methane $\left(\mathrm{CH}_{4}\right)$ flow driven by a constant pressure gradient for a wide range of temperatures and pressures. Our goal consists in overcoming the assumptions of previous studies by considering a realistic porous carbon molecular structure. In the case of CS1000a, a model based on MaxwellStefan diffusion theory ? ${ }^{?}$ coupled to a Langmuir adsorption isotherm reproduces the simulated permeabilities, thus confirming that diffusion and adsorption are the dominating transport mechanisms as long as AIMT can be neglected. However, only the results obtained for CS1000 turn out to be consistent with experimental data found in the literature, which suggests that the description of transport properties in real microporous carbons does involve strong AIMT mechanisms.

\section{Model and simulation schemes}

\section{Principles of the DCV-GCMD numerical experiment}

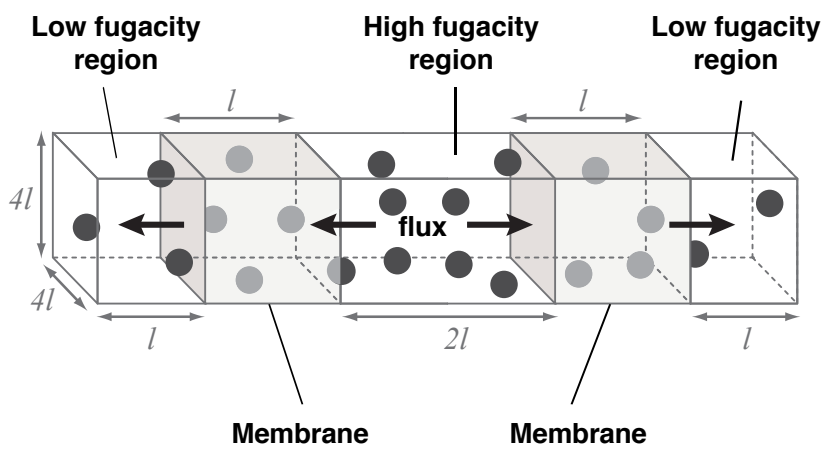

Figure 1: Schematic representation of DCV-GCMD method. In our simulations the membrane thickness stands as one CS1000a unit cell: $l=2.5 \mathrm{~nm}$. 
Our numerical experiment consists in reproducing an experimental set-up used for permeability measurements, as illustrated in Figure ??. For that purpose we use the DCV-GCMD method. ? ? High and low fugacity reservoirs are imposed at each end of the membranes. This allows the application of periodic boundary conditions. While the fugacities in these reservoirs are controlled by means of Grand Canonical Monte Carlo simulation, molecular motions are described using Molecular Dynamics simulation. Once the system has reached the steady-state regime, the molar flux $J$ is estimated by counting the number of molecules $N$ crossing the membrane of cross section $S$ during a time interval $\Delta t$ :

$$
J=\frac{N}{S \Delta t}
$$

The permeability is then defined as the flux per unit of fugacity gradient $(\Delta f / l)$ across the membrane:

$$
P_{e}=\frac{J l}{\Delta f}
$$

\section{Membrane and fluid models}

As microporous membranes, we chose two models of porous carbons, CS1000a and CS1000, with main characteristics listed in Table ??. These models were obtained by Jain and co-workers ${ }^{?}$ as results of Hybrid Reverse Monte Carlo (HRMC) simulations, where arrangement of the atoms was driven by systematic refinement of simulated radial distribution functions to match X-ray scattering experimental data and simultaneous energy minimization. The pore size distributions and pair correlation functions shown in Figure ??(c) clearly indicate that both models are disordered polydisperse microporous materials. Furthermore, the CS1000 model was recently used to model $\mathrm{CH}_{4}$ and $\mathrm{CO}_{2}$ adsorption induced swelling in coal, in quantitative agreement with experimental data. ' We can therefore consider these molecular models as fairly representative of disordered microporous carbons.

In the following of this paper, we study the flow of pure $\mathrm{CH}_{4}$ through CS1000a and CS1000. The $\mathrm{CH}_{4}$ molecules, as well as the $\mathrm{CS}$ models carbon and hydrogen atoms are described as simple 
(a)

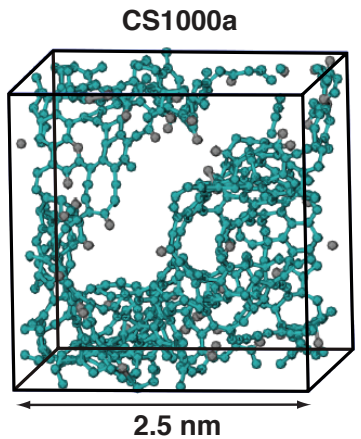

(b)
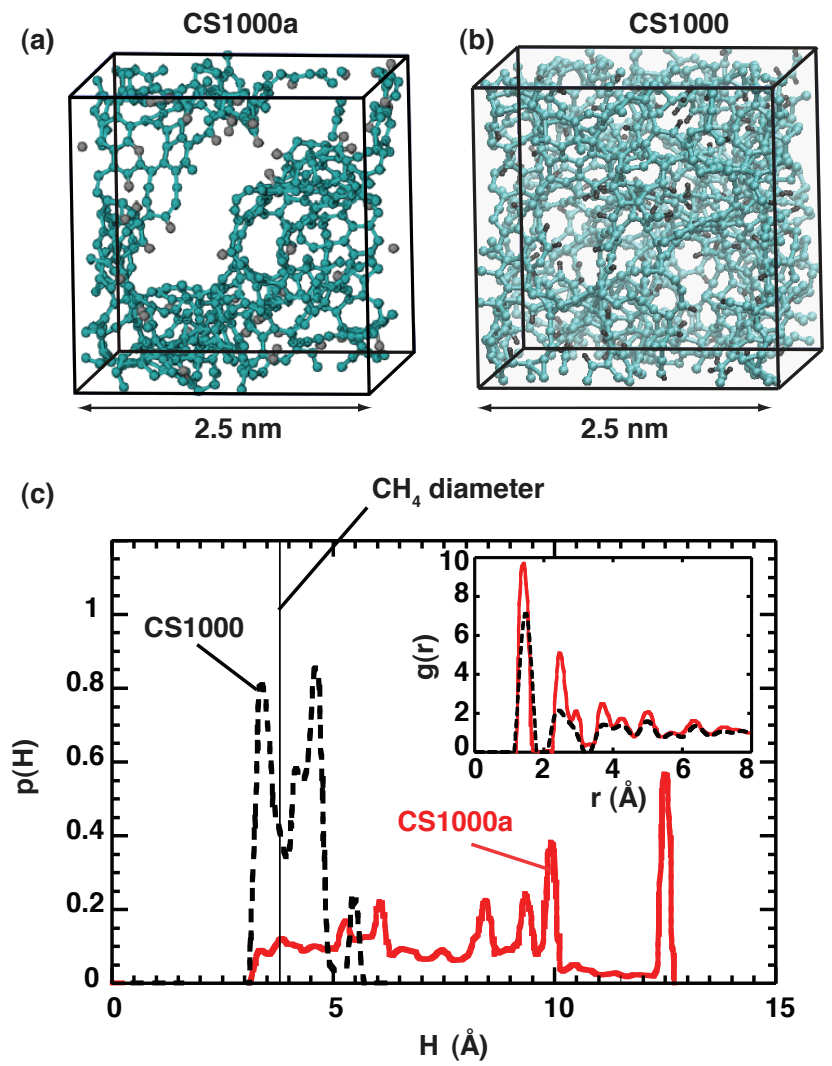

Figure 2: (a), (b) Snapshots of CS1000a and CS1000 cubic unit cells respectively (2.5 nm). Carbon and hydrogen atoms appear in cyan and grey respectively. (c) Pore size distributions of CS1000a (red solid line) and CS1000 (black dashed line). The vertical black solid line indicates the diameter of $\mathrm{CH}_{4}$ molecules. Inset figure: Radial distribution functions of CS1000a (red solid line) and CS1000 (black dashed line).

Table 1: Structural properties of CS1000a and CS1000 models.

\begin{tabular}{lll}
\hline property & CS1000a & CS1000 \\
\hline linear size $(\mathrm{nm})$ & 2.5 & 2.5 \\
carbon density $\left(\right.$ atoms $\left./ \AA^{3}\right)$ & 0.03621 & 0.0743 \\
$\mathrm{H} / \mathrm{C}$ ratio & 0.091 & 0.149 \\
pore diameter $(\mathrm{nm})$ & $0.3-1.3$ & $0.31-0.57$ \\
porosity & 0.667 & 0.088 \\
\hline
\end{tabular}


Lennard-Jones (LJ) interaction sites:

$$
U\left(r_{i j}\right)=4 \varepsilon_{i j}\left[\left(\frac{\sigma_{i j}}{r_{i j}}\right)^{12}-\left(\frac{\sigma_{i j}}{r_{i j}}\right)^{6}\right]
$$

with parameters shown in Table ??. Here $r_{i j}$ is the distance between sites $i$ and $j, \sigma_{i j}$ and $\varepsilon_{i j}$ are LJ parameters deduced from the conventional Lorentz-Berthelot combining rules. ${ }^{?}$ Because the $\mathrm{CH}_{4}$ molecule is symmetric and non-polar, its representation by a single LJ site is possible. Furthermore, the microporous adsorbent is considered infinitely rigid. Therefore, we neglect the internal degrees of freedom of the $\mathrm{CH}_{4}$ molecules as well as the many-body interactions that would arise from the flexibility of the adsorbent. Aside from quantitative corrections on the simulated diffusion coefficients and permeabilities, ${ }^{?}$ we do not expect these additional contributions to significantly change the phenomenology observed in our numerical experiments.

Table 2: Lennard-Jones potential parameters.

\begin{tabular}{lll}
\hline Site type & $\varepsilon(\mathrm{kJ} / \mathrm{mol})$ & $\sigma(\AA)$ \\
\hline methane & 1.230 & 3.73 \\
carbon & 0.232 & 3.36 \\
hydrogen & 0.125 & 2.42 \\
\hline
\end{tabular}

The DCV-GCMD set-up, described in the previous section, allowed us to investigate the permeation of $\mathrm{CH}_{4}$ through the $\mathrm{CS}$ models in the three directions of space. The permeability measured for the different spatial orientations of a given membrane was the same within a proportionality constant lower than 2. In this paper, we therefore report the results obtained for the orientation that yields intermediate values of permeability only. Furthermore, as observed in Figure ??(a), the CS1000a exhibits a $1.3 \mathrm{~nm}$ wide pore percolating through the sample. As the pore width is larger than several $\mathrm{CH}_{4}$ molecular diameters $(0.38 \mathrm{~nm})$, this guarantees that AIMT mechanisms have little influence on the overall permeability of the system and can therefore be disregarded. On the other hand, the average pore size of CS1000 $(0.41 \mathrm{~nm})$ is close to the molecular diameter of $\mathrm{CH}_{4}$ and no large pore percolates through the sample as can be seen in Figure ??(b). This means that the CS1000 structure is conducive to AIMT effects with respect to methane molecules. 


\section{Simulation details}

All simulations presented in this study are based on two types of techniques: Grand Canonical Monte Carlo simulations (GCMC) and Molecular Dynamics. 'Specifically, GCMC insertion/deletion moves are used to maintain a constant chemical potential difference between the low and high fugacity reservoirs during the DCV-GCMD simulation. The chemical potential of $\mathrm{CH}_{4}$ in the reservoirs, which is an input parameter for DCV-GCMD simulations, is estimated thanks to

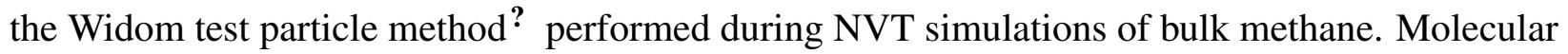
Dynamics simulations were used to move particles as no MC displacement steps were performed. The simulation box in the DCV-GCMD method (see Figure ??) is divided into five regions: two low fugacity reservoirs $5 \mathrm{~nm}$ thick (2 CS unit cells), two membranes each $2.5 \mathrm{~nm}$ thick (one CS unit cell) and one central high density reservoir $10 \mathrm{~nm}$ thick (4 CS unit cells). The transverse dimensions of the simulation box are $25 \mathrm{~nm}$ (10 unit cells) each. In order to maintain constant temperature throughout the simulation box, each region is coupled to its own (Nose-Hoover) thermostat with a damping constant of $100 \mathrm{fs}$. The temperature was then defined by subtracting the streaming velocity from the x component (flux direction) of the velocities. Typically, we imposed ten GCMD insertion or deletion steps every one MD step. The permeability calculations were run for $5 \mathrm{~ns}$ and we excluded the first $0.5 \mathrm{~ns}$ corresponding to the transient regime.

\section{Determination of the collective diffusivity and adsorption isotherm}

In addition, we performed equilibrium molecular dynamics (EMD) to calculate the collective diffusivity $D_{o}$ and adsorption isotherm of $\mathrm{CH}_{4}$ molecules in the $\mathrm{CS} 1000$ a membrane, both of which are required for the theoretical prediction of the permeability as discussed further. At the temperature $T=150{ }^{\circ} \mathrm{C}$, the collective diffusivity was found almost constant over a range of pressures $p=10-600$ bar. Fitting therefore the collective diffusivity by a constant value yields $D_{o}=(3.65 \pm 0.4) \times 10^{-8} \mathrm{~m}^{2} . \mathrm{s}^{-1}$, which is of the same order of magnitude as the data reported by Lim and Bhatia, who studied the permeation of $\mathrm{CH}_{4}$ molecules through carbon slit pores. ${ }^{\text {? }}$ For comparison, pulsed field gradient NMR experiments performed on carbon molecular sieves (CMS) 
membranes yield a typical self diffusivity of $1 \times 10^{-11} \mathrm{~m}^{2} \cdot \mathrm{s}^{-1} \cdot ?$ This difference results from the CS1000a pore size distribution, which presents significantly wider pores than usual CMS membranes materials. Simulation of the $\mathrm{CH}_{4}$ sorption isotherm in CS1000a was carried out using the DCV-GCMD technique, in which upstream and downstream fugacities were set equal to each other. This approach guarantees the fluid is adsorbed into accessible pores only. The simulated adsorption isotherm for $T=150{ }^{\circ} \mathrm{C}$ agrees with the Langmuir model (see eq ??) for the range of investigated fugacities. The best-fitting parameters are $c_{s}=10.49 \mathrm{mmol} . \mathrm{m}^{-3}$ and $b=4.52 \times 10^{-3} \mathrm{bar}^{-1}$. More details about these simulations are provided in the accompanying supplementary information.

\section{Theoretical framework}

From the thermodynamical point of view, a system of fluid particles sorbed in a immobile porous medium deviates from equilibrium when a gradient in molecules chemical potential exists. Under isothermal conditions and on the assumption that transport mechanism is diffusive, the local molar

flux $\vec{j}$ (number of mols of fluid per unit surface per unit time) through the fixed porous solid satisfies the Maxwell-Stefan equation $?$ ? :

$$
\vec{j}=-\frac{c D_{o}}{R T} \vec{\nabla} \mu
$$

where $T$ is the temperature, $R$ the ideal gas constant, $c$ the average interstitial concentration (number of moles per material unit volume), while $D_{o}$ stands as the collective diffusivity of the sorbed fluid, as previously discussed. Moreover, using the definition of the chemical potential $\mu(f, T) \equiv \mu_{0}(T)+R T \ln \left(f / f_{0}\right)$, one can alternatively consider the gradient in fugacity as the driving force of fluid motion. Hence the rearranged expression of the local molar flux:

$$
\vec{j}=-\frac{c D_{o}}{f} \vec{\nabla} f
$$

where $f$ is the fluid fugacity. In order to estimate the concentration in the microporous membrane, we refer to the classical Langmuir model, commonly used to describe adsorption isotherms of 
fluids in microporous adsorbents:

$$
c=c_{s} \frac{b f}{1+b f} .
$$

Here $c_{s}$ is the complete filling concentration and $b$ is an equilibrium adsorption constant, which can be interpreted as the inverse of a characteristic filling pressure. We stress that we use the Langmuir model for its ability to reproduce the adsorption isotherms simulated in our membrane models (see supplementary information) and its convenient analytical form. Let us now consider a microporous membrane of thickness $l$ in the $x$ direction and separating two infinite bulk fluid reservoirs exhibiting a difference in chemical potentials. Under these conditions eq ?? describes the local motion of interstitial fluid in the membrane. In an actual experiment, one can only measure the total molar flux $\vec{J}$ as a function of the fugacity drop $\Delta f$ across the membrane. Hence the averaged transport equation, obtained from the average of eq ?? along the thickness of the membrane:

$$
\vec{J}=-P_{e} \frac{\Delta f}{l} \vec{e}_{x}=-\frac{1}{l} \int_{x=0}^{x=l} d x \frac{D_{o} c}{f}\left(\frac{\partial f}{\partial x}\right) \vec{e}_{x}
$$

in which $P_{e}$ is referred to as the permeability of the membrane. It should be stressed that this definition of the permeability differs from the classical definition deriving from Darcy's law, which considers the viscous flow of a Newtonian interstitial fluid. In the present case, for the sake of generality we define the permeability as the transport coefficient relating the molar flux to the driving force of fugacity gradient, as found in the literature. ' Assuming a constant $D_{o}$, we deduce the overall permeability $P_{e}$ of the membrane from eq ?? as

$$
P_{e}=\frac{D_{o}}{\Delta f} \int_{f_{u}}^{f_{d}} d f \frac{c}{f}
$$

in which $f_{d}$ and $f_{u}$ are the downstream and upstream fugacities respectively. Finally, combining eq ?? with eq ?? the permeability is given as a function of $\Delta f$ and $f_{d}$

$$
P_{e}=\frac{D_{o} c_{s}}{\Delta f} \ln \left(1+\frac{b \Delta f}{1+b f_{d}}\right)
$$




\section{Results and Discussion}

\section{Effects of downstream pressure and pressure drop}

We investigated the permeation of methane through the CS1000a membrane for downstream pressures in the range of 0 to 300 bar at fixed temperature $T=150{ }^{\circ} \mathrm{C}$ and constant pressure drop. Figure ??(a) compares the theoretical prediction obtained for $D_{o}=3.65 \pm 0.4 \times 10^{-8} \mathrm{~m}^{2} . \mathrm{s}^{-1}$ with the permeability measured in DCV-GCMD numerical experiments according to eq ??. If the model captures the evolution of permeability with downstream pressure, we observe a systematic overestimation that can be corrected upon multiplying eq ?? by an adjusted proportionality coefficient equal to 0.85 . Accounting for the uncertainty from the estimation of $D_{o}$ during EMD simulations, the overestimation of the permeability by the theory falls in the range of $4 \%$ to $23 \%$. We attribute this discrepancy to AIMT involved in the narrowest pores of the CS1000a structure. As a result, some fluid particles present in the membrane are trapped in the smallest pores and do not contribute to the molar flux as opposed to the assumption leading to eq ??. However, while AIMT occurs in the narrowest pores, the permeability of the CS1000a is mostly driven by the diffusion and adsorption in the widest percolating pore, which explains the fair agreement with the theory.

Furthermore, in Figure ??(a) the permeability of CS1000a and CS1000 are plotted versus downstream pressure. Vieira-Linhares and Seaton report permeabilities as high as $2 \times 10^{4}$ Barrers. However, usual values of $\mathrm{CH}_{4}$ permeabilities in real disordered microporous carbon membranes range from 0.1 to $1 \times 10^{3}$ Barrers. ${ }^{?}$ ? The simulated permeabilities of CS1000a therefore exceed the experimental data by one to two orders of magnitude. This result is not surprising: the thickness of the simulated membrane $(l=2.5 \mathrm{~nm})$ is comparable to the size of the widest pore $(1.3 \mathrm{~nm})$, the latter providing a percolating path for the fluid molecules. As a result, simulated permeabilities are significantly higher than measurements performed on $15 \mathrm{~nm}$ to $2 \mu \mathrm{m}$ thick microporous carbon membranes in which no percolating micropore should be observed. On the other hand, the permeabilities obtained for CS1000, if somewhat high, are consistent with experimental data found in the literature. This result suggests that AIMT cannot be neglected in actual experiments. For 
(a)

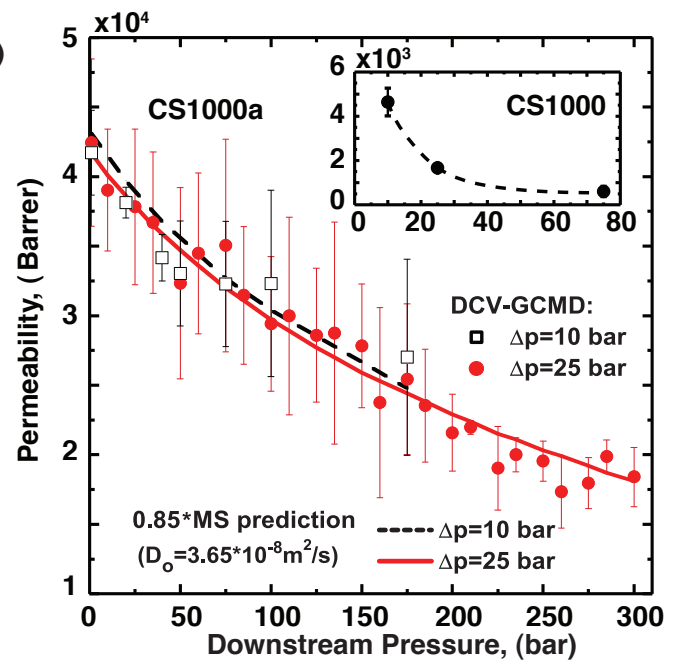

(b)

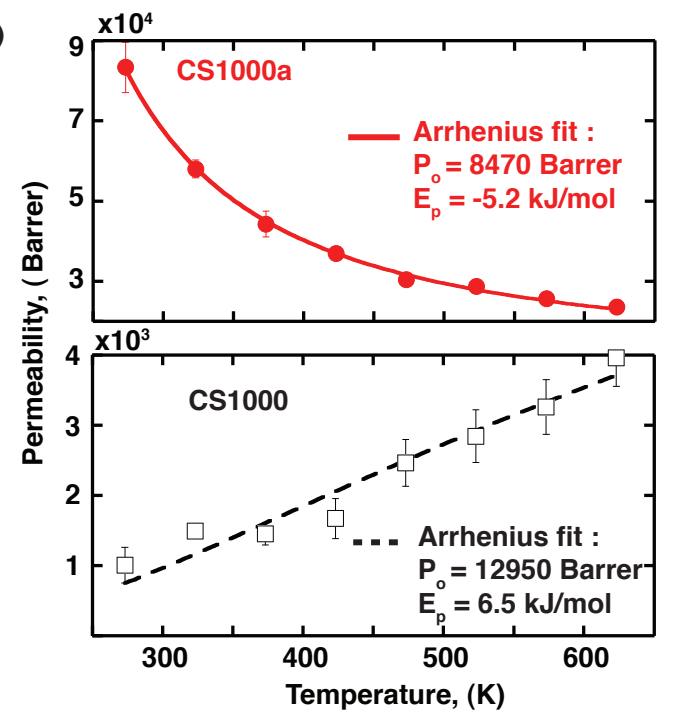

Figure 3: (a) Downstream pressure dependence of the permeability of CS1000a at $T=150{ }^{\circ} \mathrm{C}$ for two fixed pressure gradients: $\Delta p=10$ bar (black hollow squares, dashed black line) and $\Delta P=$ 25 bar (red dots, solid red line). The lines stand for the theoretical predictions (eq ??) with a correction factor adjusted to 0.85 . Inset: data obtained for CS1000 in the same conditions and $\Delta P=$ 25 bar. The dashed line is a guide for the eye. (b) Temperature dependence of the permeability of CS1000a (red dots, solid red line) and CS1000 (black hollow squares, dashed line) for $p_{u}=50$ bar and $p_{d}=25$ bar. The lines show the fits of the Arrhenius equation (see eq ??). 
both models, we observe a monotonic decrease of the permeability with downstream pressure as already observed with slit pores models. ? ? ? While the same behavior was observed for CMS

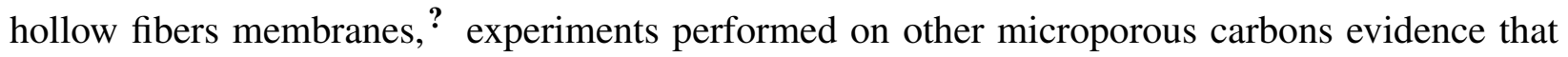
the evolution of permeability with pressure strongly depends on the nature of the sorbed fluid, more precisely when AIMT becomes important. ${ }^{?}$ Furthermore, we observe no significant effect of the pressure drop on the permeability of CS1000a in the conditions of the simulations. Noting that the typical order of magnitude of $b \Delta f \simeq 10^{-2}-10^{-1}$ is small compared to unity, from eq ?? we deduce the following approximation of the permeability:

$$
P_{e} \simeq \frac{b D_{o} c_{s}}{1+b f_{d}}
$$

which is indeed independent of $\Delta f$, and therefore independent of the pressure drop across the membrane. In addition, the model predicts a permeability decaying to zero for very high reservoir pressures. However, the range of reservoir pressures investigated in the present work did not allow us to observe this behavior.

\section{Effect of temperature}

Figure ??(b) illustrates the typical effect of temperature on methane permeability, for fixed upstream and downstream reservoir pressures, set to $p_{u}=50$ bar and $p_{d}=25$ bar respectively. Depending on the membrane model we find different trends: permeation of $\mathrm{CH}_{4}$ molecules decreases with temperature for CS1000a, whereas we observe the opposite behavior with CS1000. Classically, the temperature dependency of membranes permeability is described with an empirical Arrhenius law:

$$
P_{e}=P_{0} \exp \left(-\frac{E_{p}}{R T}\right)
$$

where $E_{p}$ is the permeation activation energy. The fit by the empirical Arrhenius law reported in Figure ??(b) yields a negative permeation activation energy $E_{p}=-5.2 \mathrm{~kJ} \cdot \mathrm{mol}^{-1}$ for CS1000a. This result is consistent with other numerical studies on graphitic slit pores, ${ }^{?}$ but contradicts nu- 
merous experiments performed on real microporous carbons. On the contrary, CS1000 exhibits a positive activation energy $E_{p}=+6.5 \mathrm{~kJ} \cdot \mathrm{mol}^{-1}$ in good agreement with experimental data found in the literature. ${ }^{?}$ This result further demonstrates that the AIMT mechanism, also referred to as the pore constriction or pore mouth effect by some authors, ${ }^{?} ?$ does stand as the limiting process in actual permeation experiments. ? ? As a result of AIMT, fluid particles diffusion is confined to local areas of the membrane. Therefore, fluid molecules need to overcome energy barriers in order to permeate through the constrictions and explore adjacent pores. Consecutively, rising the temperature increases the flux by releasing trapped molecules in the flow, which is consistent with models based on transition state theory. ${ }^{?}$ In the case of CS1000a such effects do not prevail, which explains the decrease of permeability with temperature as further discussed.

\section{Discussion on the diffusive/adsorptive regime}

Table 3: Estimation of the contribution from temperature and pressure on the permeability of CS1000a. $P_{e}^{*}$ was computed assuming the molar flux is given by the product of the interstitial fluid concentration $c$ and their mean velocity $v$ in the flow direction.

\begin{tabular}{lllll}
\hline $\mathrm{T}(\mathrm{C})$ & $p_{d}(\mathrm{bar})$ & $\mathrm{c}\left(\mathrm{kmol} / \mathrm{m}^{3}\right)$ & $v(\mathrm{~m} / \mathrm{s})$ & $P_{e}^{*}($ Barrer $)$ \\
\hline 50 & 150 & 6.868 & 1.9 & 62420 \\
50 & 25 & 2.391 & 8.4 & 73263 \\
350 & 25 & 0.379 & 18.9 & 16139 \\
\hline
\end{tabular}

Regarding the CS1000a, we observed the monotonic decrease of the permeability of the membrane with respect to the reservoir pressures and temperature. We can intuitively understand this behavior by arguably neglecting the effect of AIMT mechanisms and considering the influence of adsorption and diffusion only. Because of the importance of fluid adsorption in the microporous membrane, the two main contributions to the flux of fluid particles through the microporous membrane, namely the interstitial fluid concentration $c$ and the average transport velocity $\vec{v}$, are intertwined. For instance, as adsorption weakens with increasing temperature or reducing pressure, the interstitial concentration decays, which in turn favors the mobility of the fluid molecules. Reducing the temperature or raising the reservoir pressure triggers the exact opposite mechanism. 
Table ?? reports the permeability $P_{e}^{*}$ resulting from the approximation $J \approx c v$, for a fixed pressure drop $\Delta p=25$ bar and different values of reservoir pressures and temperatures. This approximation qualitatively reproduces the direct measurements reported in Figure ??. For a given pressure drop $\Delta p$, we can describe the influence of reservoir pressures and temperature on permeability as follows:

1. At fixed temperature, upon increasing the reservoir pressures the loss in mobility of the fluid particles is more important than the gain in interstitial concentration. Consecutively, the permeability decreases with reservoir pressure. Data from Table ?? obtained at $T=50{ }^{\circ} \mathrm{C}$ illustrates well this behavior.

2. If the temperature is raised while the pressures in each reservoirs are held constant, the loss in concentration is greater than the gain in particle mobility, thus leading to a decrease of the permeability, as observed for the data obtained at $p_{d}=25$ bar and reported in Table ??.

\section{Conclusion}

In this study, we have used DCV-GCMD to investigate pure methane permeation through two molecular models of disordered microporous carbon membranes, namely CS1000a and CS1000. In the case of CS1000a, we identified the decrease of interstitial fluid molecules mobility and concentration as the two main mechanisms responsible for the decay of permeability with pressure and temperature respectively. This phenomenology was already observed with simpler slit pores models and is characteristic of purely diffusive/adsorptive permeation as AIMT can be disregarded. A theoretical model based on the simplified Maxwell-Stefan equation and the Langmuir adsorption isotherm indeed captures the evolution of permeability with respect to reservoirs pressures. In the case of CS1000, which exhibits significantly narrower pores along with more tortuous flow paths, AIMT comes into play. As a result, the permeability of CS1000 increases with temperature consistently with most of the experiments performed on real microporous carbons. 


\section{Acknowledgement}

The authors thank Shell and Schlumberger for financial support through the MIT X-Shale research project. Romain Vermorel acknowledges financial support from the Fulbright Program through the Fulbright Aquitaine Program.

\section{Supporting Information Available}

"Estimation of the collective diffusivity and adsorption isotherm by means of equilibrium molecular dynamics simulations, investigation of the influence of the ratio of MC to MD steps in DCV-GCMD simulations." This material is available free of charge via the Internet at http: //pubs.acs.org/. 


\section{Graphical TOC Entry}

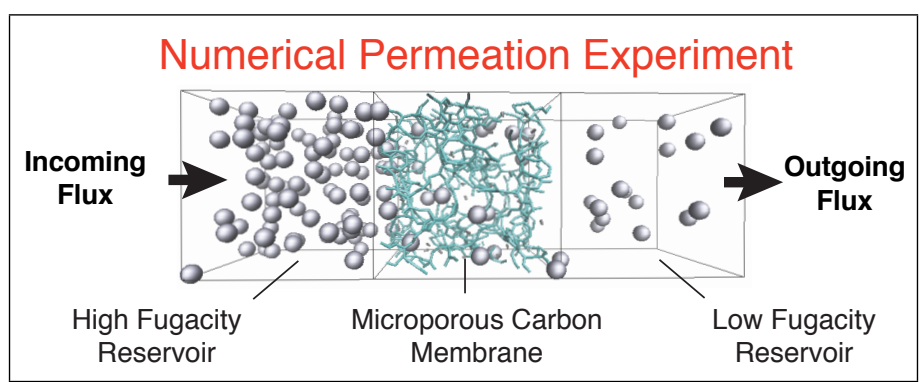

